\title{
Measurement of blood flow in myocardial layers: A step toward comprehensive physiological evaluation
}

\author{
Kartik Gupta, MBBS, ${ }^{\text {a }}$ Fadi G. Hage, MD, ${ }^{\text {b,c }}$ Jonathan McConathy, MD PhD, ${ }^{d}$ \\ and Navkaranbir S. Bajaj, MD, MPH ${ }^{\mathrm{b}, \mathrm{c}, \mathrm{d}}$ \\ a Department of Internal Medicine, All India Institute of Medical Sciences, New Delhi, India \\ b Division of Cardiovascular Disease, University of Alabama at Birmingham, Birmingham, AL \\ c Section of Cardiology, Birmingham Veterans Affair Medical Center, Birmingham, AL \\ d Division of Molecular Imaging and Therapeutics, Department of Radiology, University of \\ Alabama at Birmingham, Birmingham, AL
}

Received Nov 12, 2018; accepted Nov 13, 2018

doi: $10.1007 / \mathrm{s} 12350-018-01533-7$

See related article, pp. 1665-1674

Widely used single-photon emission computed tomography-myocardial perfusion imaging (SPECTMPI) lacks spatial resolution to identify myocardial layers as well as the ability to measure absolute myocardial blood flow (MBF). ${ }^{1}$ The pitfalls of relative MPI using SPECT are well known in terms of underestimating the extent of coronary artery disease (CAD). ${ }^{2,3}$ These shortcomings have been overcome by cardiac positron emission tomography (PET) which quantifies absolute global MBF. Quantification of absolute MBF at stress and rest, and the derivation of myocardial flow reserve (MFR) is more desirable than relative flow assessment due to its diagnostic utility, powerful prognostic nature, improved accuracy, reproducibility, and simplified post-processing. ${ }^{1,4-8}$ Recent consensus statement from the American Society of Nuclear Cardiology and the Society of Nuclear Medicine and Molecular Imaging Cardiovascular Council indicates that these measures are at the cusp of translation into clinical practice. ${ }^{7}$ While it is well known that there is a transmural variation in $\mathrm{MBF}$ in $\mathrm{CAD}$, wherein the subendocardium (SEN) is more vulnerable to

Reprint requests: Navkaranbir S. Bajaj, MD, MPH, Division of Cardiovascular Disease, University of Alabama at Birmingham, Birmingham, AL; nbajaj@uabmc.edu

J Nucl Cardiol 2020;27:1675-8.

$1071-3581 / \$ 34.00$

Copyright (c) 2018 American Society of Nuclear Cardiology. ischemia than the subepicardium $(\mathrm{SEP})^{9,10}$, the majority of cardiac PET literature has focused on measurement of average blood flow across the entire myocardial thickness rather than in layers. This is likely due to the complexities involved in making those measurements. However, if performed accurately, these measurements will reflect the true heterogeneous nature of ischemia and may have incremental diagnostic and prognostic value.

Over the past several decades, various techniques have been used to evaluate MBF in layers. The earliest studies, using radionuclide-labeled microspheres to measure regional MBF in animals, suggested a preferential increase in the blood flow to the SEP layer relative to the SEN layer in case of stenosis. ${ }^{9-11}$ Several studies utilizing different modalities including cardiac PET, magnetic resonance (MR), and computed tomography (CT) perfusion have demonstrated transmural variation in MBF in patients with CAD (Table 1). ${ }^{12-14}$ While it has been shown in these studies that it may be feasible to make these measurements using different modalities, the diagnostic utility of these measurements has not been well established. Current literature is conflictive in the added value of measuring MBF in layers: while MR assessment of MBF measurements in layers suggests a higher accuracy for CAD detection ${ }^{15,16}$, CT and $\left[{ }^{15} \mathrm{O} \mathrm{H}_{2} \mathrm{O}\right.$ PET studies do not show a similar advantage over global MBF measurements. ${ }^{17,18}$

Although transmural variation in MBF has been demonstrated by cardiac PET using $\left[{ }^{15} \mathrm{O}\right] \mathrm{H}_{2} \mathrm{O}$ in patients with $\mathrm{CAD}^{17}$, in this issue of the journal, Sciagrà et al. ${ }^{19}$ for the first time explored the feasibility and utility of $\left[{ }^{13} \mathrm{~N} \mathrm{NH}_{3}\right.$ cardiac PET imaging for quantifying variations in MBF in separate layers of the myocardium using 


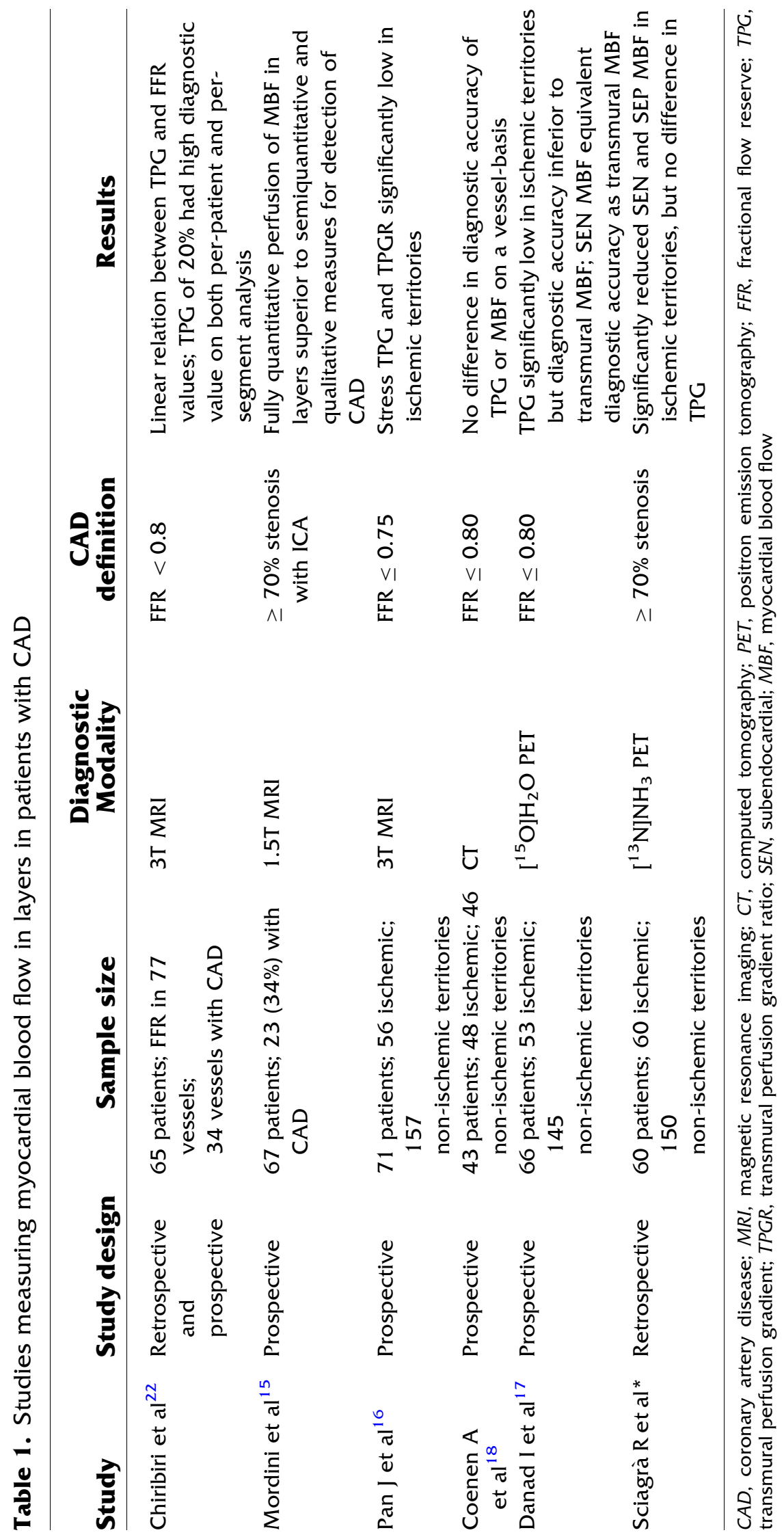


an automated procedure in patients with known or suspected CAD.

Sciagrà et al. ${ }^{19}$ studied 70 patients who underwent $\left[{ }^{13} \mathrm{~N}\right] \mathrm{NH}_{3}$ cardiac PET imaging, of whom 36 patients had significant $\mathrm{CAD}$ (defined as stenosis $\geq 70 \%$ on invasive coronary angiography) with 60 ischemic territories. Those authors did not observe any difference in resting global, SEN, or SEP MBF among patients with or without CAD. Whereas during hyperemia, there was a significant decrease in global, SEN, and SEP MBF in patients with versus those without CAD. Similar results were obtained when data were analyzed on a vascular territory basis. However, the measurement of MBF in layers did not show improved diagnostic accuracy above whole thickness measures. Whole thickness MFR and SEN MFR were significantly lower among patients with $\mathrm{CAD}$, whereas hyperemic transmural perfusion gradient (TPG: the ratio of SEN MBF/SEP MBF) was not different between patients with or without CAD. They also demonstrate acceptable intraobserver correlation for ten randomly selected cases.

The current study differs in some respects from another cardiac PET study done by Danad et al. ${ }^{17}$, where $\left[{ }^{15} \mathrm{O}\right] \mathrm{H}_{2} \mathrm{O}$ was used for quantification of MBF in layers in patients with an intermediate likelihood of CAD (defined as fractional flow reserve $\leq 0.80$ ). In the study by Danad et al. ${ }^{17} \mathrm{MBF}$ measures had similar directional results as observed by Sciagrà et al. ${ }^{19}$ However, they observed a lower hyperemic TPG in patients with significant $C A D$ versus those without, which was not observed by Sciagrà et al. ${ }^{19}$ They also reported not only good intraobserver (like Sciagrà et al. ${ }^{19}$ ) but also reasonable interobserver correlation while making these measurements.

Taken together, both these studies suggest that measurement of MBF in layers in patients with CAD using cardiac PET is feasible. Both studies were done at experienced cardiac PET centers and demonstrate that more physiological MBF measurements are possible. However, the implementation of this knowledge beyond research realms may not have reached primetime yet. The amount of activity in the blood pool at the time of MBF measurement as well as differences in resolution related to positron ranges of different PET radionuclides will likely be the weighing factors in implementing these approaches. Furthermore, MBF measurements in layers are subject to similar pitfalls as global $\mathrm{MBF}$ measurements, including variability related to use of different software, lack of standardization in image acquisition/reconstruction protocols, and use of different radionuclides and scanners. ${ }^{20}$ There is also lack of data for normal $\mathrm{MBF}$ in layers using different radiotracers and the incremental diagnostic and prognostic utility of these measures.
This study paves the way to quantify MBF in layers using a cardiac PET with several caveats and questions which require further exploration:

1. Demonstration of intra- and interobserver variability of these measurements or lack thereof in multicenter studies.

2. Standardization of radiotracers, acquisition, reconstruction, kinetic modeling, and post-processing tools for making these measurements to reduce variability.

3. Assessment of variability introduced by the spatial resolution of cardiac PET, partial volume averaging, spillover, and other artifacts while making these measurements

4. Demonstration of the incremental value of transmural $\mathrm{MBF}$ variation in CAD patients beyond whole thickness measurements and possible ways to incorporate this information in making clinical decisions.

5. Demonstration of the diagnostic and prognostic values of these measurements in non-CAD diseases, including left ventricular hypertrophy, aortic stenosis, hypertrophic cardiomyopathy, and coronary microvascular disease. ${ }^{21}$

6. Comparison with MR and CT perfusion techniques in terms of accuracy, feasibility, radiation dose, cost, and incremental diagnostic value.

In conclusion, this innovative study should encourage further investigations to make a measurement of $\mathrm{MBF}$ in layers more reliable and clinically useful.

\section{Disclosure}

Kartik Gupta and Jonathan McConathy have no disclosures. Fadi G. Hage is supported by Astellas Pharma, Novartis Pharma and GE Healthcare. Navkaranbir S. Bajaj is supported by American College of Cardiology Presidential Career Development Award and National Center for Advancing Translational Research of the National Institutes of Health under award number UL1TR001417.

\section{References}

1. Bengel FM. Leaving relativity behind: Quantitative clinical perfusion imaging. J Am Coll Cardiol 2011;58:749-51.

2. Lima RS, Watson DD, Goode AR, et al. Incremental value of combined perfusion and function over perfusion alone by gated SPECT myocardial perfusion imaging for detection of severe three-vessel coronary artery disease. J Am Coll Cardiol 2003;42:64-70.

3. Berman DS, Kang X, Slomka PJ, et al. Underestimation of extent of ischemia by gated SPECT myocardial perfusion imaging in patients with left main coronary artery disease. J Nucl Cardiol 2007;14:521-8

4. Bajaj NS, Osborne MT, Gupta A, et al. Coronary microvascular dysfunction and cardiovascular risk in obese patients. J Am Coll Cardiol 2018;72:707-17. 
5. Osborne MT, Bajaj NS, Taqueti VR, et al. Coronary Microvascular Dysfunction Identifies Patients at High Risk of Adverse Events Across Cardiometabolic Diseases. J Am Coll Cardiol 2017;70:2835-7.

6. Bravo PE, Bergmark BA, Vita T, et al. Diagnostic and prognostic value of myocardial blood flow quantification as non-invasive indicator of cardiac allograft vasculopathy. Eur Heart $\mathrm{J}$ 2018;39:316-23.

7. Murthy VL, Bateman TM, Beanlands RS, et al. Clinical Quantification of Myocardial Blood Flow Using PET: Joint Position Paper of the SNMMI Cardiovascular Council and the ASNC. J Nucl Med 2018;59(2):273-93.

8. Gupta A, Taqueti VR, van de Hoef TP, et al. Integrated Noninvasive Physiological Assessment of Coronary Circulatory Function and Impact on Cardiovascular Mortality in Patients With Stable Coronary Artery Disease. Circulation 2017;136:2325-36.

9. Bache RJ, Ball RM, Cobb FR, Rembert JC, Greenfield JC Jr. Effects of nitroglycerin on transmural myocardial blood flow in the unanesthetized dog. J Clin Invest.1975;55:1219-28.

10. Hoffman JI. Transmural myocardial perfusion. Prog Cardiovasc Dis 1987;29:429-64.

11. Hoffman JI. Determinants and prediction of transmural myocardial perfusion. Circulation 1978;58:381-91.

12. Cheirif J, Zoghbi WA, Bolli R, O’Neill PG, Hoyt BD, Quinones MA. Assessment of regional myocardial perfusion by contrast echocardiography. II. Detection of changes in transmural and subendocardial perfusion during dipyridamole-induced hyperemia in a model of critical coronary stenosis. J Am Coll Cardiol 1989;14:1555-65.

13. Keijer JT, van Rossum AC, Wilke N, et al. Magnetic resonance imaging of myocardial perfusion in single-vessel coronary artery disease: implications for transmural assessment of myocardial perfusion. J Cardiovasc Magn Reson 2000;2:189-200.
14. George RT, Arbab-Zadeh A, Miller JM, et al. Adenosine stress 64and 256-row detector computed tomography angiography and perfusion imaging: a pilot study evaluating the transmural extent of perfusion abnormalities to predict atherosclerosis causing myocardial ischemia. Circ Cardiovasc Imaging 2009;2:174-82.

15. Mordini FE, Haddad T, Hsu LY, et al. Diagnostic accuracy of stress perfusion CMR in comparison with quantitative coronary angiography: fully quantitative, semiquantitative, and qualitative assessment. JACC Cardiovasc Imaging 2014;7:14-22.

16. Pan J, Huang S, Lu Z, et al. Comparison of myocardial transmural perfusion gradient by magnetic resonance imaging to fractional flow reserve in patients with suspected coronary artery disease. Am J Cardiol 2015;115:1333-40.

17. Danad I, Raijmakers PG, Harms HJ, et al. Impact of anatomical and functional severity of coronary atherosclerotic plaques on the transmural perfusion gradient: a [15O]H2O PET study. Eur Heart J 2014;35:2094-105.

18. Coenen A, Lubbers MM, Kurata A, et al. Diagnostic value of transmural perfusion ratio derived from dynamic CT-based myocardial perfusion imaging for the detection of haemodynamically relevant coronary artery stenosis. Eur Radio. 2017;27:230916.

19. Sciagrà Roberto, Giubbini Raffaele, Kubik Tomasz, et al. Subendocardial and Sub-epicardial Measurement of Myocardial Blood Flow Using 13NH3 PET in Man. J Nucl Cardiol 2018.

20. Bajaj Navkaranbir S., Hage Fadi G., McConathy Jonathan, et al. Myocardial blood flow measures using cardiac positron emission tomography: Software comparisons. J Nucl Cardiol 2018.

21. Klocke FJ, Lee DC. Probing transmural myocardial perfusion with CMR. JACC Cardiovasc imaging 2014;7:23-5.

22. Chiribiri A, Hautvast GL, Lockie T, et al. Assessment of coronary artery stenosis severity and location: quantitative analysis of transmural perfusion gradients by high-resolution MRI versus FFR. JACC Cardiovasc Imaging 2013;6:600-9. 